\title{
Health care utilisation of infants with chronic lung disease, related to hospitalisation for RSV infection
}

A Greenough, S Cox, J Alexander, W Lenney, F Turnbull, S Burgess, P A J Chetcuti, N J Shaw, A Woods, J Boorman, S Coles, J Turner

\begin{abstract}
Aims-To compare the use of health care resources and associated costs between infants with chronic lung disease (CLD) who had or had not an admission with a proven respiratory syncytial virus (RSV) infection.

Methods-Review of community care, outpatient attendances, and readmissions in the first two years after birth. Patients: 235 infants (median gestational age 27 weeks) evaluated in four groups: 45 infants with a proven RSV admission (RSV proven); 24 with a probable bronchiolitis admission; 60 with other respiratory admissions; and 106 with non-respiratory or no admissions.

Results-The RSV proven compared to the other groups required more frequent and longer admissions to general paediatric wards and intensive care units, more outpatient attendances and GP consultations for respiratory related disorders, and had a higher total cost of care.

Conclusion-RSV hospitalisation in patients with CLD is associated with increased health service utilisation and costs in the first two years after birth. (Arch Dis Child 2001;85:463-468)
\end{abstract}

Keywords: premature infants; chronic lung disease; respiratory syncytial virus

Respiratory syncytial virus (RSV) is the most important respiratory pathogen in childhood. Ninety per cent of children will have at least one significant RSV infection in the first two years after birth. In the United States alone it accounts for more than 90000 paediatric hospitalisations and 4500 deaths annually. A humanised RSV monoclonal antibody (palivizumab) is now available. In a large randomised trial, ${ }^{1}$ prophylaxis with palivizumab was associated with a significantly lower hospitalisation rate in infants born prematurely with or without chronic lung disease (CLD). It has been argued $^{2-4}$ that prophylaxis with palivizumab may not be cost effective, except perhaps in infants with CLD receiving supplementary oxygen. ${ }^{3}$ Those studies, ${ }^{2-4}$ however, only took into account the cost of care related to the initial hospitalisation and are likely to have underestimated the financial burden, as RSV infection results in long term respiratory morbidity. Several prospective follow up studies ${ }^{5-7}$ have shown that even $7-11$ years after the initial RSV infection, previously healthy babies born at term are more likely to wheeze, cough, and require antiasthma treatment than carefully matched controls. Certain groups, including those born prematurely, especially if they had CLD, are at risk of developing severe RSV disease. ${ }^{8-10}$ As a consequence, it seems likely that RSV infection would particularly impact unfavourably on the long term pulmonary outcome of CLD infants, with important implications for resource utilisation. Unfortunately such data, essential for the planning of provision, are not available from a UK based population. The aim, therefore, of this study was to evaluate the use of health care resources and associated cost of treatment of RSV infection, both in the hospital and in the community of infants who were born prior to 32 weeks of gestational age and developed CLD.

\section{Methods}

The study sample consisted of neonates born at less than 32 weeks of gestational age who had been admitted during the first week after their birth to one of four neonatal intensive care units between 1 July 1994 and 1 July 1997 and subsequently developed CLD (defined as an oxygen dependency beyond 28 days after birth). A retrospective review was made of their care in the community and during any readmission following discharge from the neonatal intensive care unit until the children were 2 years of age. The study was approved by the local research ethics committee of each of the four hospitals.

In each hospital, a research sister reviewed the neonatal units' admission books to identify infants born prior to 32 weeks of gestational age. The medical records of the infants were then reviewed to ascertain whether they fulfilled the eligibility criteria-that is, they were born at less than 32 weeks of gestation, developed CLD, and survived until discharge. The general practitioners (GPs) of the infants who fulfilled the criteria were then contacted to determine whether the infants were still alive and to confirm their home address. The parents of surviving infants were then sent information about the study and asked to send back written consent if they agreed for their child's hospital and primary care medical records to be examined. On obtaining consent a unique study identifier was given to each child. In all subsequent reports and communications, each patient was only identified by their unique study identifier.

From the neonatal admission, the following data were retrieved: birth weight; use of antenatal steroids and postnatal surfactant; 
development of an airleak (pneumothorax/ pulmonary interstitial emphysema) or patent ductus arteriosus (clinical diagnosis with or without echocardiographic confirmation); duration of ventilatory support and supplementary oxygen; and use of high frequency oscillation and/or nitric oxide. From the GPs' records for each child the following data were retrieved: venue of all hospital readmissions; number of GP consultations; all medication prescribed; use and duration of home oxygen; number of referrals to a health visitor or community paediatric nurse; and use of community support services. For each hospital admission the following information was recorded: diagnosis or symptoms leading to the admission; duration of stay; whether the child was admitted to a paediatric ward, high dependency (HDU), or intensive care unit (ICU); days of supplementary oxygen and intravenous fluids; surgical or therapeutic procedures; and duration and frequency of all medication. Each infant's hospital records were examined to ascertain the number of outpatient attendances.

Costs were assessed over the two year period. The costs per bed/day were obtained from the four main hospitals in the study. The mean cost ( $£ 634$ per day HDU and $£ 1380$ per ICU) was used in the calculation of the cost of stay. The cost of each admission to HDU or ICU was calculated by multiplying the number of nights by the cost per bed/day, to which was added the cost of any surgical or therapeutic procedure. The cost of admission to a general paediatric ward was based on the data displayed on the NHS website (The New NHS 1999 Reference Costs). Drug costs were calculated from the British National Formulary prices. The cost of attendance as an outpatient was estimated, assuming 15 minutes with a consultant paediatrician and using the mean of the outpatient costs of the four main hospitals. The cost of care by a GP was estimated, assuming an 8.4 minute consultation at the surgery $(£ 18$ per consultation). The cost of a GP's time was based on average net remuneration, allowing for capital costs and overheads. The cost of a domicilary visit by community staff was estimated, assuming a 20 minute consultation. The cost of domicilary visits for health visitors, paediatric nurses, and oxygen nurse specialists was based on average net remuneration for specialist nurses, allowing for superannuation, national insurance, travel, and capital overheads ( $£ 27$ per visit). All visits to practice nurses or routine visits to health visitors, for example for immunisations, were not recorded as these were considered the usual costs for infants. The costs of care were summarised under six headings: primary care total, primary care respiratory related, primary care drugs, hospital drugs, hospital stay, and outpatient attendance.

STATISTICAL ANALYSIS

The infants were divided into four groups according to whether they had had at least one hospital admission associated with a proven RSV infection (RSV proven); an admission with probable bronchiolitis (probable bronchiolitis); an admission because of other respiratory problems (other respiratory); or either admissions for only non-respiratory problems or no admissions (non-respiratory). RSV infection was considered proven only if the RSV antigen was identified in a nasopharyngeal aspirate. The diagnosis of probable bronchiolitis was based on clinical signs including fever, dyspnoea, and wheeze. Differences between the four groups were assessed for statistical significance using a Kruskal-Wallis nonparametric analysis of variance and either a $\chi^{2}$ or Fisher's exact test as appropriate for discrete data. Pairwise comparisons were made if significant deviation was found. Similar analyses were also performed after dividing the groups into those who were or were not discharged home on supplementary oxygen. The age at which each child in the RSV proven group was first hospitalised because of RSV infection was noted. Analysis was then undertaken to determine whether hospital resource utilisation and associated costs of care were greater in the RSV proven group following that admission compared to the other three groups throughout the study period.

\section{PATIENTS}

The medical records of 1581 infants were screened. One hundred and eighty one of the infants had died. Other reasons for exclusion from the study were: admittance to the neonatal intensive care unit only after the first week after birth $(n=35)$, absence of CLD $(n=906)$, and the medical records could not be found $(n=200)$. Written consent was obtained from 240 of the 259 sets of parents of eligible infants. It was not possible to obtain primary care and/or hospital data on five of the 240 infants. Analysis, therefore, was performed on the data from 235 infants who had a median gestational age of 27 weeks (range 22-31) and birth weight of $934 \mathrm{~g}$ (range 510-3000).

\section{Results}

During their first two years after birth, 45 infants $(19 \%)$ had at least one hospital admission for a proven RSV infection, 24 had at least one admission for probable bronchiolitis, 64 had admissions with other respiratory problems, and 106 either had admissions for non-respiratory problems or were never admitted. The demographic features and neonatal course of the proven RSV group did not differ significantly from any of the other three groups, with the exception of the duration of ventilation on the neonatal ICU (NICU) and the time spent in the NICU (table 1).

After discharge from the NICU, the 235 children had in total 560 hospital admissions. The 45 children who had at least one admission for RSV proven infection had a total of 242 admissions: 55 were for an RSV proven infection, 13 for probable bronchiolitis, and 174 for other reasons. The median admission rate per infant for all causes differed significantly between the groups, being highest in the proven RSV group than the other three groups $(\mathrm{p}<0.001 ;$ table 2$)$. The total days spent in 
Table 1 Demographics and neonatal course according to RSV status

\begin{tabular}{lllll}
\hline & $\begin{array}{l}\text { RSV proven } \\
(n=45)\end{array}$ & $\begin{array}{l}\text { Probable bronchiolitis } \\
(n=24)\end{array}$ & $\begin{array}{l}\text { Other respiratory } \\
(n=60)\end{array}$ & $\begin{array}{l}\text { Non-respiratory } \\
(n=106)\end{array}$ \\
\hline $\begin{array}{l}\text { Gestational age (weeks) } \\
\text { Birth weight (g) }\end{array}$ & $26(24-31)$ & $26(23-31)$ & $27(22-31)$ & $27(24-31)$ \\
Antenatal steroids & $863(640-2178)$ & $834(590-1538)$ & $972(630-1696)$ & $960(510-3000)$ \\
Postnatal surfactant & 81 & 93 & 88 & 86 \\
CPAP & 91 & 88 & 90 & 84 \\
IPPV & 54 & 46 & 51 & 64 \\
HFOV & 98 & 100 & 98 & 95 \\
NO & 5 & 4 & 10 & 3 \\
Airleak & 2 & 29 & 5 & 11 \\
PDA & 11 & 46 & 40 & 31 \\
Postnatal dexamethasone & 38 & 50 & 38 & 36 \\
Duration of IPPV (days) & 41 & $4.5(0-62)$ & $10(0-103)^{\star}$ & $7(0-96)$ \\
Duration of admission (days) & $12(0-58)^{\star}$ & $83(18-260)$ & $76(10-464)^{\star \star}$ & $66(2-827)$ \\
Home oxygen & $42(16-360)^{\star \star}$ & 25 & 52 & 30 \\
\hline
\end{tabular}

Data are presented as median (range) or (\%).

${ }^{\star} \mathrm{p}<0.05,{ }^{\star \star} \mathrm{p}<0.01$

CPAP, continuous positive airway pressure; IPPV, intermittent positive pressure ventilation; HFOV, high frequency oscillatory ventilation; NO, nitric oxide; PDA, patent ductus arteriosus.

hospital and in the general paediatric wards per infant also differed significantly between the four groups (table 3), being longest in the RSV proven group $(\mathrm{p}<0.01)$.

Twelve infants in the proven RSV group were admitted to ICU (nine had one admission and three two admissions) compared to two in the probable bronchiolitis group, six in the other respiratory group, and only one in the non-respiratory group. ICU admission rates differed significantly between the groups, with the rate being highest in the proven RSV group (table 3). Days spent in ICU per infant also differed significantly between the groups, being highest in the RSV proven group (table 2). Outpatient attendances per infant during the first two years of life were significantly higher in the RSV proven group ( $\mathrm{p}<0.05$; table 4 ), as were primary care consultations with GPs for respiratory illnesses ( $\mathrm{p}<0.05$; table 2$)$.

The mean total cost of care during the first two years after birth, following discharge from the NICU differed significantly between the four groups (table 2), being highest in the RSV proven group $(p<0.01)$. The total cost of inpatient care, hospital stay, hospital drugs, and outpatient attendances differed significantly between the four groups, being highest in the RSV proven group. Although total primary care costs did not differ significantly between the four groups, costs related to consultations for respiratory illness and for drug prescriptions did differ significantly, the highest costs occurring in the proven RSV group (table 5).

If only the children who were discharged from the NICU on supplementary oxygen at home were considered, the RSV proven group had a higher total cost of care than the other groups $(\mathrm{p}<0.001)$. This related to higher costs associated with hospital stay $(\mathrm{p}<0.01)$ and primary care drugs $(p<0.001)$. Consideration of only the children who were not discharged home on oxygen revealed that the RSV proven compared to the other groups generated higher costs of care in all areas considered $(\mathrm{p}<0.05)$, except for primary care drugs.

Table 2 Health care utilisation and cost of care related to RSV status

\begin{tabular}{|c|c|c|c|c|}
\hline & $\begin{array}{l}R S V \text { proven } \\
(n=45)\end{array}$ & $\begin{array}{l}\text { Probable bronchiolitis } \\
(n=24)\end{array}$ & $\begin{array}{l}\text { Other respiratory } \\
(n=60)\end{array}$ & $\begin{array}{l}\text { Non-respiratory } \\
(n=106)\end{array}$ \\
\hline Hospital admission rate per infant & $\begin{array}{l}5.4(4) \\
{[1-20]}\end{array}$ & $\begin{array}{l}2.8(2) \\
{[1-11]}\end{array}$ & $\begin{array}{l}3.1(3) \\
{[1-14]}\end{array}$ & $\begin{array}{l}0.6(0) \\
{[0-5]}\end{array}$ \\
\hline Days in hospital ${ }^{\star}$ & $\begin{array}{l}39.8(21) \\
{[4-282]}\end{array}$ & $\begin{array}{l}17.7(6.5) \\
{[1-70]}\end{array}$ & $\begin{array}{l}16.3(8) \\
{[1-114]}\end{array}$ & $\begin{array}{l}2.2(0) \\
{[0-25]}\end{array}$ \\
\hline Days in ICU* & $\begin{array}{l}2.9(0) \\
{[0-38]}\end{array}$ & $\begin{array}{l}0.9(0) \\
{[0-13]}\end{array}$ & $\begin{array}{l}0.5(0) \\
{[0-16]}\end{array}$ & $\begin{array}{l}0(0) \\
{[0-1]}\end{array}$ \\
\hline Outpatient attendances ${ }^{\star \star}$ & $\begin{array}{l}11.9(12) \\
{[2-27]}\end{array}$ & $\begin{array}{l}8.3(8) \\
{[1-30]}\end{array}$ & $\begin{array}{l}9.2(8) \\
{[0-36]}\end{array}$ & $\begin{array}{l}8.6(7.5) \\
{[0-41]}\end{array}$ \\
\hline $\begin{array}{l}\text { Consultantsion with GP for respiratory } \\
\text { illnesses } \star \star \star\end{array}$ & $\begin{array}{l}8.3(7) \\
{[0-28]}\end{array}$ & $\begin{array}{l}7(6.5) \\
{[0-26]}\end{array}$ & $\begin{array}{l}8.5(6) \\
{[0-48]}\end{array}$ & $\begin{array}{l}5.6(5) \\
{[0-25]}\end{array}$ \\
\hline $\begin{array}{l}\text { Total cost of care } \\
\text { Presented as mean }(95 \% \mathrm{CI})\end{array}$ & $\begin{array}{l}12638 \\
(8041 ; 17235)\end{array}$ & $\begin{array}{l}6059 \\
(3427 ; 8690)\end{array}$ & $\begin{array}{l}5683 \\
(3427 ; 6775)\end{array}$ & $\begin{array}{l}2461 \\
(2074 ; 2849)\end{array}$ \\
\hline
\end{tabular}

Data are presented as mean (median) and [range] unless otherwise stated. Between group difference: ${ }^{\star} \mathrm{p}<0.001,{ }^{\star \star} \mathrm{p}<0.01,{ }^{\star \star \star} \mathrm{p}<0.05$.

Table 3 Frequency and duration of admissions (days) according to RSV status

\begin{tabular}{|c|c|c|c|c|}
\hline & $\begin{array}{l}\text { RSV proven } \\
(n=45)\end{array}$ & $\begin{array}{l}\text { Probable bronchiolitis } \\
(n=24)\end{array}$ & $\begin{array}{l}\text { Other respiratory } \\
(n=60)\end{array}$ & $\begin{array}{l}\text { Non-respiratory } \\
(n=106)\end{array}$ \\
\hline Hospital admission rate per infant ${ }^{\star}$ & $\begin{array}{l}5.4(4) \\
{[1-20]}\end{array}$ & $\begin{array}{l}2.8(2) \\
{[1-11]}\end{array}$ & $\begin{array}{l}3.1(3) \\
{[1-14]}\end{array}$ & $\begin{array}{l}0.6(0) \\
{[0-5]}\end{array}$ \\
\hline Days in hospital ${ }^{\star}$ & $\begin{array}{l}39.8(21) \\
{[4-282]}\end{array}$ & $\begin{array}{l}17.7(6.5) \\
{[1-70]}\end{array}$ & $\begin{array}{l}16.3(8) \\
{[1-114]}\end{array}$ & $\begin{array}{l}2.2(0) \\
{[0-25]}\end{array}$ \\
\hline Days in ICU* & $\begin{array}{l}2.9(0) \\
{[0-38]}\end{array}$ & $\begin{array}{l}0.9(0) \\
{[0-13]}\end{array}$ & $\begin{array}{l}0.5(0) \\
{[0-16]}\end{array}$ & $\begin{array}{l}0(0) \\
{[0-1]}\end{array}$ \\
\hline Days in paediatric ward ${ }^{\star}$ & $\begin{array}{l}30.7(16) \\
{[2-239]}\end{array}$ & $\begin{array}{l}13.9(4.5) \\
{[0-57]}\end{array}$ & $\begin{array}{l}12.4(6.0) \\
{[0-100]}\end{array}$ & $\begin{array}{l}1.6(0) \\
{[0-22]}\end{array}$ \\
\hline
\end{tabular}

Data are presented as mean (median) and [range].

Between group difference: ${ }^{\star} \mathrm{p}<0.001$. 
Table 4 Outpatient attendances and primary care contacts by RSV status

\begin{tabular}{lllll}
\hline & $\begin{array}{l}R S V \text { proven } \\
(n=45)\end{array}$ & $\begin{array}{l}\text { Probable bronchiolitis } \\
(n=24)\end{array}$ & $\begin{array}{l}\text { Other respiratory } \\
(n=60)\end{array}$ & $\begin{array}{l}\text { Non-respiratory } \\
(n=106)\end{array}$ \\
\hline Outpatient attendances $^{\star}$ & $11.9(12)$ & $8.3(8)$ & $9.2(8)$ & $8.6(7.5)$ \\
GP contacts & {$[2-27]$} & {$[1-30]$} & {$[0-36]$} & $10-41]$ \\
Community care contacts & $16.3(14)$ & $12.5(12)$ & $18.3(13)$ & $14.6(13)$ \\
& {$[0-56]$} & {$[1-37]$} & {$[0-76]$} & {$[0-44]$} \\
Consultations with GP for respiratory illness ${ }^{\star \star}$ & $28.2(21)$ & $16.4(16)$ & $24.4(17)$ & $18.7(16)$ \\
& {$[1-169]$} & {$[2-37]$} & {$[0-125]$} & {$[1-69]$} \\
\end{tabular}

Data are presented as mean (median) and [range].

Between groups difference: ${ }^{\star} \mathrm{p}<0.01,{ }^{\star \star} \mathrm{p}<0.05$

Table 5 Cost of care (in pounds sterling) by RSV status

\begin{tabular}{lllll}
\hline & RSV proven & Probable bronchiolitis & Other respiratory & Non-respiratory \\
\hline Primary care drugs $^{\star}$ & $663(432 ; 903)$ & $340(132 ; 548)$ & $513(371 ; 654)$ & $269(180 ; 359)$ \\
Primary care respiratory consultations $^{\star \star}$ & $182(137 ; 226)$ & $155(97 ; 212)$ & $191(145 ; 236)$ & $117(101 ; 134)$ \\
Total primary care cost & $626(359 ; 893)$ & $339(250 ; 429)$ & $507(359 ; 654)$ & $378(326 ; 430)$ \\
Hospital stay ${ }^{\star \star}$ & $9347(5192 ; 13916)$ & $4127(1646 ; 6607)$ & $3409(2480 ; 4339)$ & $717(429 ; 1096)$ \\
Hospital drugs ${ }^{\star \star \star}$ & $340(186 ; 494)$ & $61(17 ; 104)$ & $69(42 ; 96)$ & $7(3 ; 12)$ \\
Total inpatient cost $t^{\star \star \star}$ & $9687(5459 ; 13916)$ & $4187(1668 ; 6705)$ & $3478(2530 ; 4427)$ & $725(435 ; 1015)$ \\
Outpatient attendance & $149 \star(1119 ; 1871)$ & $1024(596 ; 1453)$ & $980(802 ; 1159)$ & $953(822 ; 1084)$ \\
Total cost of care & $12638(8041 ; 17235)$ & $6059(3427 ; 8690)$ & $5683(3427 ; 6775)$ & $2461(2074 ; 2849)$ \\
\hline
\end{tabular}

Data are presented as mean $(95 \% \mathrm{CI})$.

Between groups difference: ${ }^{\star} \mathrm{p}<0.005,{ }^{\star \star} \mathrm{p}<0.02,{ }^{\star \star \star} \mathrm{p} p 0.001$

The mean age of the 45 infants at their first admission for an RSV proven infection was 7.9 months (median 6.6, range 1-22). This was the first hospital admission for 16 of the 45 infants. After their first RSV proven admission, that group had a median total number of admissions of 2 (range 1-17) for a median total duration of 13 days (range 2-164); 10 of the children were admitted to ICU on 12 occasions for a median duration of 0 days (range 0-38). Their health care utilisation after their first RSV proven admission was significantly greater than that of the other three groups throughout the entire study period. Similarly, the inpatient costs (mean $£ 7294$, range $£ 768-62$ 552) were significantly higher in the RSV proven group, considering the costs accrued only after the initial RSV proven hospitalisation compared to the costs generated by the other three groups throughout the study period $(\mathrm{p}<0.001)$.

\section{Discussion}

This study has shown that CLD patients who require hospitalisation for RSV infection have significantly greater morbidity than those without RSV infection, as highlighted by more frequent and longer duration admissions to paediatric wards and ICUs. The criteria for selection of patients into the study were chosen to include those at highest risk of the adverse consequences from RSV infection. Infants born prior to 32 weeks of gestation are disadvantaged because of abnormal airways and/or a relative or complete lack of maternal RSV neutralising antibody. ${ }^{11}$ The more severe impact of RSV infection on premature infants who develop CLD is highlighted by their hospitalisation rate of $19 \% .{ }^{12}$ We diagnosed CLD as oxygen dependency beyond 28 days as that definition has been frequently used in studies assessing the impact of RSV infection. ${ }^{12}$ In addition, in the present population of preterm infants, oxygen dependency beyond 28 days is a sensitive and specific predictor of chronic respiratory morbidity. ${ }^{13} 14$
We divided the patients into four groups as we suspected that a clinical diagnosis of bronchiolitis in children with a high rate of underlying respiratory problems might be relatively inaccurate in a retrospective study. Our hypothesis was supported by the finding that the RSV proven group had a significantly different outcome to the probable bronchiolitis group, who fared similarly to the other respiratory group. These results underscore the importance, when prospectively trying to accurately assess the impact of RSV infection in infants with an underlying respiratory disorder, of establishing the diagnosis by examination of a nasopharyngeal aspirate.

The incidence of hospitalisation for RSV infection among preterm infants in North America varies from $2.8 \%$ to $37 \%$, higher rates being experienced in those with underlying cardiac or pulmonary disease and those oxygen dependent beyond 28 days. $^{2}$ The hospitalisation rate for proven RSV infection among our CLD infants of $19 \%$ was higher than the $17.4 \%$ reported in the PREVENT study ${ }^{12}$ or the $12.8 \%$ in the IMpact study, ${ }^{1}$ but lower than the $38.8 \%$ recently noted. ${ }^{15}$ The PREVENT and IMpact studies only reported data from a single RSV season, whereas we recorded the outcome during a two year period. The longer period of follow up was chosen as we wished to document the full impact of RSV infection, and it is well appreciated that some young children will be readmitted on more than one occasion with proven RSV infection. ${ }^{16}$ Although in older children and adults, reinfections with RSV are less severe with each successive occurrence, ${ }^{17}$ this is not so with young children requiring supplementary oxygen. ${ }^{18}$ In such patients, the risk for severe disease remains high, even with their second and third infection, and persists while the child's lung disease is sufficiently severe that they require supplementary oxygen. ${ }^{18}$ In a recently reported retrospective cohort study, ${ }^{15}$ it was shown that children with CLD had high rates of RSV hospitalisation until 24 
months of age, whereas after the first year of life, children with congenital heart disease or prematurity have rates no higher than that of children at low risk who were less than 12 months old. In a study which has followed a group of CLD infants less than 32 weeks of gestational age for two years, a hospitalisation rate of $45 \%$ was documented. Our 69 patients in the RSV proven and probable bronchiolitis groups had a total of 94 admissions for proven or suspected RSV infection during the first two years after birth.

Welliver $^{19}$ reported a $32-37 \%$ rate of ICU admission for CLD patients, $17-25 \%$ requiring mechanical ventilation. The rates in the PREVENT study (4.6\% admission and 2\% ventilation) and the IMpact study (3\% admission and $0.2 \%$ ventilation) were much lower. ${ }^{12}$ In the present population $5 \%$ of children with RSV proven infection were admitted to ICU.

We have shown that those CLD patients who had a proven RSV infection generated significantly higher costs of care, approximately three times, of those who were RSV negative (table 5). Previous estimations of the cost of care for RSV patients have usually only included the costs related to hospitalisation. ${ }^{3} \mathrm{One}^{2}$ did include the cost of attendance in casualty or an acute clinic immediately preceding the admission and non-medical costs such as those incurred by the parents because of loss of work. Marchetti and colleagues ${ }^{20}$ examined data on hospital charges (including outpatient costs) related to RSV infection in infants less than 35 weeks of gestational age with or without CLD. Assuming a hospitalisation rate of $12.8 \%$, they calculated the expected hospital charge would vary widely, from $\$ 1341$ to $\$ 21327$, depending on the source used. The lower figure was based on a multicentre assessment of infants regardless of comorbidities, ${ }^{21}$ and the higher figure based on an assessment of high risk infants in a single institution. ${ }^{22}$ The costs, however, were only calculated for a single season, which is likely to be an underestimate of the impact of RSV infection, ${ }^{18}$ and they ignored certain types of health service utilisation also likely to generate extra cost. ${ }^{23}$ The Avon perinatal follow up study has shown that preterm compared to term infants are not only on average six times more likely to be readmitted and require more outpatient attendances, but have more frequent GP consultations and see their health visitors more often for non-routine visits. ${ }^{23}$ We therefore felt that if the true cost of care for RSV infection was to be obtained, it was essential to record not only hospital admissions and outpatient attendances, but also GP consultations and visits from other community staff over a two year period. This highlighted that CLD patients with proven RSV infection generated higher costs for all aspects of care; the differences in cost compared to the other groups were statistically significant, not only when considering costs related to hospital admissions and drugs, but also outpatient attendances and primary care respiratory related consultations and drugs.

The RSV proven group differed significantly from the other groups in requiring significantly

\section{Key message}

- Prematurely born CLD infants who are hospitalised with RSV infection have an increased health service utilisation in the first two years after birth

longer durations of neonatal ventilation and NICU stay. This might suggest they had suffered more severe neonatal lung disease and hence required hospitalisation when suffering an RSV infection. The four groups, however, did not differ with regard to other indicators of respiratory disease severity - that is, the proportions suffering complications such as pneumothorax or requiring postnatal dexamethasone. In addition, the proportion of the RSV proven group discharged home on supplementary oxygen did not differ similarly from the other three groups. The four groups were also similar with respect to their gestational age and birth weight. Our data highlight the difficulty of predicting from their neonatal characteristics which CLD infants are likely to suffer severe consequences of RSV infections. Research to further refine methods for identifying infants at high risk is desirable in order to facilitate targeting of RSV prophylaxis.

The patients were discharged from the neonatal units at different times of the year and thus their ages varied at the beginning of the RSV season. Hence, the mean age at the first RSV proven hospitalisation was approximately 8 months (range 1-22 months). The latter figure emphasises that CLD patients may still experience serious consequences of RSV infection even in the second year after birth. ${ }^{18}$ As the patients were on average 8 months of age when they experienced their first RSV proven hospitalisation episode, their health care utilisation and associated costs only after, but not before, that age could be attributed to RSV infection. Thus, to obtain a more accurate estimate of the extra care attributable to RSV infection, we compared health care utilisation and associated costs in the RSV proven group post initial RSV hospitalisation to the other three groups for the whole study period. Although we were assessing the RSV proven group over a shorter time period than the other groups, the former group still had a significantly greater hospitalisation requirement, with significantly higher inpatient, outpatient, and primary care costs.

We conclude that RSV infection associated with hospital admission in very preterm infants with CLD causes important morbidity, as indicated by their significantly higher use of health service resource. Our study indicates that the current estimations of the cost effectiveness of RSV prophylaxis ${ }^{24}$ are weakened by an incomplete consideration of the longer term costs and health impacts of RSV infection. At this time, however, there are no data available regarding the impact of prophylaxis on the long term sequelae of RSV infection. Future research needs to take full account of significant cost offsets that may be available through appropriate prophylaxis and 
the quality of life impact of RSV on the infants and their families.

The research nurses were funded by Abbott Laboratories Ltd We are grateful to $\mathrm{Mr} \mathrm{M}$ Elsley for assistance with data analysis and Ms S Williams for secretarial assistance.

1 Anonymous. Palivizumab, a humanized respiratory syncytial virus monoclonal antibody, reduces hospitalization from respiratory syncytial virus infection in high-risk infants. The IMpact-RSV Study Group. Pediatrics 1998;102(3, part 1):531-7.

2 Joffe S, Escobar GJ, Black SB, et al. Rehospitalization for respiratory syncytial virus among premature infants. Pediatrics 1999;104(4 part 1):894-9.

3 Thomas M, Bedford-Russell A, Sharland M. Hospitalisation for RSV infection in ex-preterm infants-implications for use of RSV immune globulin. Arch Dis Child 2000;83:122-7.

4 Clark SJ, Beresford W, Subhedar NV, Shaw NJ. Respiratory syncytial virus infection in high risk infants and the potential impact of prophylaxis in a United Kingdom cohort. Arch Dis Child 2000;83:313-16.

5 Sigurs N, Bjarnason R, Sigurbergsson F, Kjellman B. Respiratory syncytial virus bronchiolitis in infancy is an ratory syncytial virus bronchiolitis in infancy is an important risk factor for asthma and al

Respir Crit Care Med 2000;161:1501-7.
6 Noble V, Murray M, Webb MSC, et al. Respiratory status and allergy nine to 10 years after acute bronchiolitis. Arch and allergy nine to 10 years

7 Stein RT, Sherrill D, Morgan WJ, et al. Respiratory syncytial virus in early life and risk of wheeze and allergy by age 13 years. Lancet 1999;354:541-5.

8 Cunningham CK, McMillan JA, Gross SJ. Rehospitalization for respiratory illness in infants of less than 32 weeks' gestation. Pediatrics 1991;88:527-32.

9 Groothuis JR, Gutierrez KM, Lauer BA. Respiratory syncytial virus infection in children with bronchopulmonary dysplasia. Pediatrics 1988;82:199-203.

10 Wang EE, Law BJ, Robinson JL, et al. PICNIC (Pediatric Investigators Collaborative Network on Infections in Canta) study of the role of a and respiratory syncytial Canada) study of the role of age and respiratory syncytial virus neutralizing antibody on respiratory syncytial virus 1llness in patients with
Pediatrics 1997;99:e9.

11 Meissner CD, Welliver RC, Chartrand SA, et al. Prevention of respiratory syncytial virus infection in high risk infants: consensus opinion on the role of immunoprophylaxis with respiratory syncytial virus hyperimmune globulin. Pediatr Infect Dis f 1996;15:1059-68.

12 The PREVENT study group. Reduction of respiratory syncytial virus hospitalization among premature infants and infants with bronchopulmonary dysplasia using respiratory syncytial virus immune globulin prophylaxis. Pediatrics 1997;99:93-9.

13 Kinali M, Greenough A, Dimitriou G, Yuksel B. Chronic respiratory morbidity following premature deliveryprediction of prolonged respiratory support requirement. Eur F Pediatr 1999;158:493-6.

14 Palta M, Sadek M, Barnet JH, et al. Evaluation of criteria for chronic lung disease in surviving very low birthweight infants. F Pediatr 1998;132:57-63.

15 Boyce TG, Mellen BG, Mitchel EF, et al. Rates of hospitalization for respiratory syncytial virus infection among zation for respiratory syncytial virus infection
children in Medicaid. $₹$ Pediatr 2000;137:865-70.

16 Prober CG, Sullender WM. Advances in prevention of respiratory syncytial virus infections. F Pediatr 1999;135:546-58.

17 Glezen WP, Taber LH, Frank AL, Kasel JA. Risk of primary infection and reinfection with respiratory syncytial virus. Am $\mathcal{F}$ Dis Child 1986;140:543-6.

18 Groothuis JR, Salbenblatt CR, Lauer RA. Severe respiratory syncytial virus infection. Am F Dis Child 1990;144:346-8.

19 Welliver RC. Respiratory syncytial virus immunoglobulin and monoclonal antibodies in the prevention and treatment of respiratory syncytial virus infection. Semin Perinatol 1998;22:87-95.

20 Marchetti A, Lau H, Magar R, et al. Impact of palivizumab on expected costs of respiratory syncytial virus infection in preterm infants: potential for savings. Clin Ther 1999;21 752-66.

21 Grier CE, Howe BJ. Economic impact of pneumonia due to RSV infection. Presented at ICAAC 35th Annual Meeting, 17-20 Sept, San Francisco, California. ICAAC: San Francisco, California, 1995.

22 Oelburg D, Reininger M, van Eeckout J. A cost benefit analysis of respiratory syncytial virus hyperimmune globulin (RSV-IVIG) in high risk infants. Neonatal Intensive Care 1998:29-33.

23 Emond A, Evans J-A, Howat P. The continuing morbidity and use of health services by preterm infants after discharge from hospital. Ambulatory Child Health 1997;3 $121-9$

24 Sharland M, Bedford-Russell A. Preventing respiratory syncytial virus bronchiolitis. BMf 2001;322:62-3.

\section{Commentary}

Prescribers of paediatric respiratory drugs have faced some difficult decisions in the past few years. First we had alfa dornase for cystic fibrosis, costing a cool £7108 (\$10 306) per annum, then as we were recovering from that shock, we had tobramycin for nebulisation at $£ 10038$ (\$14 555) per annum (basic NHS prices as at June 2001). Barely had the ink dried on the cheques for these new drugs, than we were asked to advise on the use of palivizumab, an anti-respiratory syncytial virus (RSV) monoclonal antibody designed to protect vulnerable babies from the ravages of acute bronchiolitis. At a basic NHS price of £25444235 (\$3688-6140) for the six injections generally used to cover the RSV season, this was the final straw for many of us, and use of the product has varied widely around the country. This variation is not based on rational decision making. Rather, it reflects local experience and finances, illuminated by inadequate information on the costs and benefits of using palivizumab.

Greenough et al highlight the inadequacies of some previous studies of the costs of RSV infection in infants with chronic lung disease (CLD), and make a valiant attempt to remedy these by examining the total health care costs of such infants. In addition to the short term costs of hospitalisation associated with RSV infection, which have already been looked at, they have evaluated in great detail the financial impact of the longer term clinical deterioration that can follow RSV infection, and from which it can take many months to recover.

These figures will be of great interest to neonatologists and other paediatricians, as well as to public health doctors responsible for footing the bill for palivizumab. The latter will be quick to point out that there is no information on the effects of palivizumab on longer term disability from RSV infection, and may use this as a delaying tactic to keep their finances in balance. The sooner such information becomes available, the better.

Finally, it can reasonably be asked why there is so much emphasis on the cost efficiency of screening programmes and immunisations. In strict financial terms, very few medical and surgical treatments have been shown to be cost effective, but we don't let our patients suffer for the sake of a few pounds. So, in studies evaluating the total costs and benefits of palivizumab in babies with pre-existing CLD, we must be sure to examine clinical as well as financial outcomes. I for one would much rather prescribe a drug because it was likely to help my patient, than because it brought a smile to an accountant's face.

GEORGE RUSSELL Department of Child Health, University of Aberdeen, UK 